\title{
Research Article \\ Pricing Variance Swaps for Stochastic Volatilities with Delay and Jumps
}

\author{
Anatoliy Swishchuk ${ }^{\mathbf{1}}$ and $\mathbf{L i} \mathbf{X u}^{\mathbf{2}}$ \\ ${ }^{1}$ Mathematical and Computational Finance Laboratory, Department of Mathematics and Statistics, \\ University of Calgary, 2500 University Drive NW, Calgary, AB, Canada T2N 1N4 \\ ${ }^{2}$ H. Milton Stewart School of Industrial and Systems Engineering, Georgia Institute of Technology, \\ 765 Ferst Drive NW, Atlanta, GA 30332, USA
}

Correspondence should be addressed to Anatoliy Swishchuk, aswish@ucalgary.ca

Received 16 December 2010; Revised 9 March 2011; Accepted 16 March 2011

Academic Editor: Kambiz Farahmand

Copyright (C) 2011 A. Swishchuk and L. Xu. This is an open access article distributed under the Creative Commons Attribution License, which permits unrestricted use, distribution, and reproduction in any medium, provided the original work is properly cited.

\begin{abstract}
We study the valuation of the variance swaps under stochastic volatility with delay and jumps. In our model, the volatility of the underlying stock price process not only incorporates jumps, which are found to be active empirically, but also exhibits past dependence: the behavior of a stock price right after a given time $t$ depends not only on the situation at $t$ but also on the whole past (history) of the process $S(t)$ up to time $t$ as well. The jump part in our model is finally represented by a general version of compound Poisson processes. We provide some analytical closed forms for the expectation of the realized variance for the stochastic volatility with delay and jumps. We also present a lower bound for delay as a measure of risk. As applications of our analytical solutions, a numerical example using SEP60 Canada Index (1998-2002) is then provided to price variance swaps.
\end{abstract}

\section{Introduction}

Variance swaps are forward contracts on future realized variance, the square of the realized volatility, which provide an easy way for investors to gain exposure to the future realized variance of the asset returns instead of directly exposure to the underlying assets. The market for such derivatives develops quickly after the collapse of LCTM in 1998 when the volatilities increased to an unprecedented high level and many investors are now interested in these derivatives to hedge volatility. Recently, several papers address the valuation of variance swaps or other volatility derivatives (see [1-6]). The most recent ones are [7, 8]. Zhu and Lian [7] presented a highly efficient approach to price variance swaps with discrete sampling times. They found a closed-form exact solution for the partial differential equation system based on Heston's two-factor stochastic volatility model. The effects of jumps and 
discrete sampling on volatility and variance swaps have been investigated in [8]. For realistic contract specifications and model parameters, they found that the effect of discrete sampling is typically small while the effect of jumps can be significant.

It is known that the assumption of constant volatility in Black-Scholes model [9] is incompatible with some empirical study in the real market. Several stochastic volatility models (see $[10,11])$ are developed and able to fit some important characteristics in the market-like skews and smirks. Kazmerchuk et al. [12, 13] proposed a stochastic volatility model assuming that the volatility $\sigma\left(t, S_{t}\right)$ depends on $S_{t}=S(t+\theta)$ for $\theta \in[-\tau, 0]$, namely, stochastic volatility with delay. In [13], the Black-Scholes formula for security markets with delayed response was found, and, in [12], the continuous-time GARCH model for stochastic volatility with delay was proposed and studied. In [14], the stochastic volatility model with delay to price variance swaps was discussed and some analytical close forms for expectation of the realized continuously sampled variance were found both in stationary regime and in general case. The key features of this model are the following: (i) continuous-time analogue of discrete-time GARCH model, (ii) mean reversion, (iii) containing the same source of randomness as stock price, (iv) completeness of the market, (v) incorporating the expectation of log-return. However, there are two ways to develop this model and make it more realistic. One way is to consider multifactor stochastic volatility with delay which is developed in [15]. The other way is to consider stochastic volatility with delay and jumps which is the major work of this paper.

There are various works showing delayed response is an important factor in stock prices. Some statistical studies of stock prices (see $[16,17]$ ) indicate the dependence on past returns. For example, Kind et al. [18] obtained a diffusion approximation result for processes satisfying some equations with past-dependent coefficients, and they applied this result to a model of option pricing, in which the underlying asset price volatility depends on the past evolution to obtain a generalized (asymptotic) Black-Scholes formula. Hobson and Rogers [19] suggested a new class of nonconstant volatility models, which can be extended to include the aforementioned level-dependent model and share many characteristics with the stochastic volatility model. The volatility is nonconstant and can be regarded as an endogenous factor in the sense that it is defined in terms of the past behavior of the stock price. This is done in such a way that the price and volatility form a multidimensional Markov process. Chang and Youree [20] studied the pricing of a European contingent claim for the $(B, S)$-securities markets with a hereditary price structure in the sense that the rate of change of the unit price of the bond account and rate of change of the stock account $S$ depend not only on the current unit price but also on their historical prices. The price dynamics for the bank account and that of the stock account are described by a linear functional differential equation and a linear stochastic functional differential equation, respectively. They show that the rational price for a European contingent claim is independent of the mean growth rate of the stock. Later, Chang and Youree [21] generalized the celebrated Black-Scholes formula to include the $(B, S)$-securities market with hereditary price structure. Clearly related to our work is the work by Mohammed et al. [22] devoted to the derivation of a delayed Black-Scholes formula for the $(B, S)$-securities market using PDE approach. Hobson and Rogers [19] also observed in their past-dependent model that the resulting implied volatility is U-shaped as a function of strike price. However, they dealt with only a special case where the model can be reduced to a system of SDEs. Unfortunately, not every past-dependent model can be reduced to a system of SDEs, and a more sophisticated approach, as developed in this paper, is needed. 
Our model of stochastic volatility exhibits past dependence: the behavior of a stock price right after a given time $t$ depends not only on the situation at $t$ but also on the whole past (history) of the process $S(t)$ up to time $t$. This draws some similarities with fractional Brownian motion models due to a long-range dependence property. Our work is also based on the GARCH $(1,1)$ model (see [23])

$$
\sigma_{n}^{2}=\gamma V+\alpha \ln ^{2}\left(\frac{S_{n-1}}{S_{n-2}}\right)+(1-\alpha-\gamma) \sigma_{n-1}^{2}
$$

or, more general,

$$
\sigma_{n}^{2}=\gamma V+\frac{\alpha}{l} \ln ^{2}\left(\frac{S_{n-1}}{S_{n-1-l}}\right)+(1-\alpha-\gamma) \sigma_{n-1}^{2}
$$

and the work of Duan [24] where he showed that it is possible to use the GARCH model as the basis for an internally consistent option pricing model. If we write down the last equation in differential form, we can get the continuous-time GARCH with expectation of log-returns of zero:

$$
\frac{d \sigma^{2}(t)}{d t}=\gamma V+\frac{\alpha}{\tau} \ln ^{2}\left(\frac{S(t)}{S(t-\tau)}\right)-(\alpha+\gamma) \sigma^{2}(t)
$$

If we incorporate nonzero expectation of log-return (using Itô Lemma for $\ln (S(t) / S(t-\tau))$ ), then we arrive at our continuous-time GARCH model for stochastic volatility with delay:

$$
\frac{d \sigma^{2}\left(t, S_{t}\right)}{d t}=\gamma V+\frac{\alpha}{\tau}\left[\int_{t-\tau}^{t} \sigma\left(s, S_{s}\right) d W(s)\right]^{2}-(\alpha+\gamma) \sigma^{2}\left(t, S_{t}\right) .
$$

We should mention that in the work of Kind et al. [18], a past-dependent model was defined by diffusion approximation. In their model, the volatility depends on the quadratic variation of the process, while our model deals with more general dependence of the volatility on the history of the process over a finite interval.

In addition to delay, jumps are another evidence in the financial market. During the last decade, financial models based on jumps processes have acquired increasing popularity in risk management and option pricing applications. A good reference is [25], which provides a self-contained overview of the theoretical, numerical, and empirical aspects of using jump processes in financial modeling. Stochastic volatility models with jumps are also included in this book. Some attempts have been made to incorporate jumps in stochastic volatility to price variance and volatility swaps (see $[26,27]$ ).

The literature has mainly focused on two approaches: (1) time-varying volatility models that allow for market extremes to be outcome of normally distributed shocks that have a randomly changing variance and (2) models that incorporate discontinuous jumps in the asset price. Neither stochastic volatility models nor jump models have alone proven entirely empirically successful. For example, in the time-series literature, the models run into problems explaining large price movements such as the October $1987 \mathrm{crash}$. Hence, a price jump cannot explain the enormous increase in implied volatility following the crash of 1987. In response to these issues, researchers have proposed models that incorporate both stochastic volatility and jumps components. 
Eraker et al. [28] use returns data to investigate the performance of models with jumps in volatility using the class of jump-in-volatility models proposed by Duffie et al. [29]. The results in [28] show that the jump-in-volatility models provide a significant better fit to the returns data.

The key risk factors considered in option pricing models, besides the diffusive price risk of the underlying asset, are stochastic volatility and jumps, both in the asset price and its volatility. Models that include some or all of these factors were developed in [11, 29-32]. The importance of jumps in volatility has become apparent in recent studies, which try to explain the time series properties of both stock and option prices, for example, [28, 33]. In an asset allocation context, the main papers analyzing the impact of jumps are [34-36]. In the presence of jumps markets are incomplete and the analysis far less tractable. Technical issues aside, jumps are important because they represent a significant source of nondiversifiable risk as discussed at length in [37].

There is currently fairly compelling evidence for jumps in the level of financial prices. The most convincing evidence comes from recent nonparametric work using high-frequency data as in [38, 39] among others. Also, the paper by Todorov and Tauchen [40] conducts a nonparametric analysis of the market volatility dynamics using high-frequency data on the VIX index compiled by the CBOE and the $S \& P 500$ index. The data suggest that stock market volatility is best described as a pure jump process without a continuous component. Their results imply that a plausible model for stochastic volatility is a model of pure-jump type whose driving jumps come from a very active Lévy process.

The jumps in stock market volatility are found to be so active that this discredits many recently proposed stochastic volatility models without jumps (see $[37,40]$ ).

Another advantage of our stochastic volatility model with delay and jumps is mean-reversion: the volatility is allowed to mean revert. Such models have shown some success in modeling interest rate (e.g., [41]). The sharp decline of option implied that spot volatility following the extreme peak caused by the 1987 crash would be indicative of such a model.

In this paper, we incorporate a jump part in the stochastic volatility model with delay proposed by Swishchuk [14] to price variance swaps. We find some analytical closed forms for the expectation of the realized continuously sampled variance for stochastic volatility with delay and jumps. The jump part in our model is finally represented by a general version of compound Poisson processes, and the expectation and the covariance of the jump sizes are assumed to be deterministic functions. We note that after adding jumps, the model still keeps those good features of the previous model such as continuous-time analogue of GARCH model and mean reversion. But it is more realistic and still quick to implement. Besides, we also present a lower bound for delay as a measure of risk. As applications of our analytical solutions, a numerical example using S\&P60 Canada index (1998-2002) is also provided to price variance swaps with delay and jumps. We show that the price differences with and without jumps depend on the jump intensity of the underlying price processes, that is, how you count and estimate the number of jumps.

The rest of the paper is organized as follows. In Section 2, we introduce some basic concepts of variance swaps, and, in Section 3, we recall the pricing model of variance swaps for stochastic volatility with delay presented by Swishchuk [14]. The pricing model and analytical formulae for stochastic volatility with delay and jumps are discussed in Section 4. The main and new results are formulated in Theorems 4.1-4.6 of this section. A lower bound for delay as a measure of risk is presented in Section 5. Finally, we give a numerical example in Section 6 and conclude in Section 7. 


\section{Variance Swaps}

A variance swap is a forward contract on realized variance, the square of the realized volatility. Its payoff at expiration is equal to

$$
N\left(v_{R}-K_{\mathrm{var}}\right)
$$

where $v_{R}=\sigma_{R}^{2}$ is the realized stock variance (quoted in annual terms) over the life of the contract, $K_{\mathrm{var}}$ is the delivery price for variance, and $N$ is the notional amount of the swap in dollars per annualized volatility point squared. The holder of a variance swap at expiration receives $N$ dollars for every point by which the stock's realized variance $\sigma_{R}^{2}$ has exceeded the variance delivery price $K_{\mathrm{var}}$. We note that usually $N=\alpha I$, where $\alpha$ is a converting parameter such as 1 per volatility square and $I$ is a long-short index ( +1 for long and -1 for short).

The measure of realized variance which will be used is defined at the beginning of the contract. The continuous time realized variance over the life of the contract $T$ is generally in the following form (here $\sigma^{2}(t)$ is a stock variance):

$$
v_{R}=\sigma_{R}^{2}=\frac{1}{T} \int_{0}^{T} \sigma^{2}(s) d s,
$$

and its discrete version is defined as follows:

$$
v_{n}=\frac{n}{(n-1) T} \sum_{i=1}^{n} \log ^{2} \frac{S_{t_{i}}}{S_{t_{i-1}}}
$$

where we neglect $(1 / n) \sum_{i=1}^{n} \log \left(S_{t_{i}} / S_{t_{i-1}}\right)$ since we assume that the mean of the returns is of the order $1 / n$ and can be neglected. The scaling by $n / T$ ensures that these quantities are annualized (daily) if the maturity $T$ is expressed in years (days).

The discrete-time variance $v_{n}$ is unbiased variance estimation for $\sigma_{t}$. It can be shown that (see [4])

$$
v_{R}=\lim _{n \rightarrow+\infty} v_{n}
$$

Valuing a variance forward contract or swap is no different from valuing any other derivative security. The value of a forward contract on future realized variance with strike price $K_{\mathrm{var}}$ is the expected present value of the future payoff in the risk-neutral world:

$$
P=\mathbb{E}^{*}\left[e^{-r T}\left(v_{R}-K_{\mathrm{var}}\right)\right],
$$

where $r$ is the risk-free discount rate corresponding to the expiration date $T$ and $\mathbb{E}^{*}$ denotes the expectation under the risk-neutral measure. Since the price of entering a forward contract 
is always zero, which means it costs nothing at the beginning of the contract, and by the riskneutral pricing framework, we find that the fair delivery price of a variance swap $K_{v a r}$ is just the expectation of the realized variance under the risk-neutral probability measure:

$$
K_{\mathrm{var}}=\mathbb{E}^{*}\left[v_{R}\right]=\frac{1}{T} \int_{0}^{T} \mathbb{E}^{*}\left[\sigma^{2}(s)\right] d s,
$$

and, consequently, the value of a variance swap at some time $t$ during the contract's life given the initial specific delivery price $K$ can be expressed by

$$
P=\mathbb{E}^{*}\left[e^{-r(T-t)}\left(v_{R}-K\right)\right]=e^{-r(T-t)}\left\{\mathbb{E}^{*}\left[v_{R}\right]-K\right\} .
$$

Thus, for valuating variance swaps we only need to know $\mathbb{E}^{*}\left[v_{R}\right]$, namely, mean value of the underlying variance under the risk-neutral measure.

In this paper, we are interested in valuing variance swaps for security markets when stochastic volatility $\sigma\left(t, S_{t}\right)$ has delay response, that is, $\sigma\left(t, S_{t}\right)$ depends on $S_{t}=S(t-\tau), \tau>0$, and $S(t)$ is a stock price at time $t \in[0, T]$.

In this way,

$$
v_{R}=\frac{1}{T} \int_{0}^{T} \sigma^{2}(u, S(u-\tau)) d u, \quad \tau>0
$$

We need to calculate $\mathbb{E}^{*}\left[v_{R}\right]$ to get the fair delivery price $K_{\text {var }}$ of a variance swap, and the price of it given the specific delivery price also follows.

\section{Stochastic Volatility with Delay and without Jumps}

In this section, we recall the model and approach of pricing variance swaps for stochastic volatility with delay and without jumps presented in the paper of Swishchuk [14].

In our model, we assume that the price of the underlying asset $S(t)$ follows the following stochastic delay differential equation (SDDE):

$$
d S(t)=\mu S(t) d t+\sigma(t, S(t-\tau)) S(t) d W(t), \quad t>0,
$$

where $\mu \in \mathbb{R}$ is the mean rate of return, the volatility term $\sigma>0$ is a continuous and bounded function, and $W(t)$ is a Brownian motion on a probability space $(\Omega, \mathcal{F}, \mathbb{P})$ with a filtration $\mathcal{F}(t)$. We also let $r>0$ be the risk-free rate of return of the market.

Throughout the paper, we denote $S_{t}=S(t-\tau), t>0$ and the initial data of $S(t)$ is defined by $S(t)=\varphi(t)$, where $\varphi(t)$ is a deterministic function with $t \in[-\tau, 0], \tau>0$. 
Now we can rewrite the equation of $S(t)$ as the following expression:

$$
d S(t)=\mu S(t) d t+\sigma\left(t, S_{t}\right) S(t) d W(t)
$$

The asset volatility is defined as the solution of the following equation:

$$
\frac{d \sigma^{2}\left(t, S_{t}\right)}{d t}=\gamma V+\frac{\alpha}{\tau}\left[\int_{t-\tau}^{t} \sigma\left(s, S_{s}\right) d W(s)\right]^{2}-(\alpha+\gamma) \sigma^{2}\left(t, S_{t}\right)
$$

where the parameters $\alpha, \gamma, \tau$, and $V$ are positive constants and $0<\alpha+\gamma<1$. The Brownian motion $W(t)$ is the same as the one in (3.2).

We suppose that the following conditions are satisfied,

(C1) $\sigma\left(t, S_{t}\right)$ satisfies local Lipschitz and growth conditions;

(C2) $\int_{0}^{T} E \sigma^{2}\left(t, S_{t}\right) d t<+\infty$;

(C3) $\int_{0}^{T}\left((r-\mu) / \sigma\left(t, S_{t}\right)\right)^{2} d t<+\infty$ a.s.

Condition (C1) guarantees the existence and uniqueness of a solution of (3.1) (and (3.2)) (see [42]). Condition (C2) guarantees the existence of Itô integral in (3.3), and Condition (C3) guarantees the existence of risk-neutral measure $\mathbb{P}^{*}$ (see below).

To price the variance swaps, we need to calculate $\mathbb{E}^{*}[v]=\mathbb{E}^{*}\left[\sigma^{2}\right]$, that is, the expectation of the variance under the risk-neutral probability measure. We should find such probability measure that makes the discounted asset price $D(t)=e^{-r t} S(t)$ a local martingale and then the risk-neutral pricing formula applies.

Let $\theta(t)=(\mu-r) / \sigma\left(t, S_{t}\right)$ be the market price of risk, which is adapted to the filtration $\mathcal{F}(t)$; then by Girsanov's theorem for single Brownian motion, we obtain in the following.

(1) There is a probability measure $\mathbb{P}^{*}$ equivalent to $\mathbb{P}$ such that

$$
\frac{d \mathbb{P}^{*}}{d \mathbb{P}}=\exp \left\{-\int_{0}^{T} \theta(s) d W(s)-\frac{1}{2} \int_{0}^{T} \theta^{2}(s) d s\right\}
$$

is its Radon-Nikodym density.

(2) The discounted asset price $D(t)$ is a positive local martingale with respect to $\mathbb{P}^{*}$, and

$$
W^{*}(t)=\int_{0}^{t} \theta(s) d s+W(t)
$$

is a standard Brownian motion with respect to $\mathbb{P}^{*}$. 
Therefore, in the risk-neutral world, the underlying asset price $S(t)$ follows the process

$$
d S(t)=r S(t) d t+\sigma\left(t, S_{t}\right) S(t) d W^{*}(t)
$$

and the asset volatility is defined then as follows:

$$
\frac{d \sigma^{2}\left(t, S_{t}\right)}{d t}=\gamma V+\frac{\alpha}{\tau}\left[\int_{t-\tau}^{t} \sigma\left(s, S_{s}\right) d W^{*}(s)-(\mu-r) \tau\right]^{2}-(\alpha+\gamma) \sigma^{2}\left(t, S_{t}\right),
$$

where $W^{*}(t)$ is defined in (3.5) (see [14] for more details).

Let us take the expectations under risk-neutral measure $\mathbb{P}^{*}$ on both sides of the equation above. Denoting $v(t)=\mathbb{E}^{*}\left[\sigma^{2}\left(t, S_{t}\right)\right]$, we obtain the following deterministic delay differential equation:

$$
\frac{d v(t)}{d t}=\gamma V+\alpha \tau(\mu-r)^{2}+\frac{\alpha}{\tau} \int_{t-\tau}^{t} v(s) d s-(\alpha+\gamma) v(t)
$$

We note that this equation has stationary particular solution and approximate general solution.

In this way, we have obtained the following result in [14].

Theorem 3.1. If conditions (C1)-(C3) are satisfied, then the price of a variance swap at time t given delivery price $K$ in this case should be

$$
P \approx e^{-r(T-t)}\left[V-K+\alpha \tau \frac{(\mu-r)^{2}}{\gamma}+\left(\sigma_{0}^{2}-V-\alpha \tau \frac{(\mu-r)^{2}}{\gamma}\right) \frac{1-e^{-\gamma T}}{T \gamma}\right] .
$$

\section{Pricing Model of Variance Swaps for Stochastic Volatility with Delay and Jumps}

In the following section, we will derive some analytical closed formulae for the expectation of the realized variance for stochastic volatility with delay and jumps. First of all, we need to define the jumps and add them to the stochastic volatility model with delay. In (3.3), we find that the volatility is driven by a Brownian motion $W(t)$, but it is more realistic to consider that it is driven by a process which consists a jump part. We represent jumps in stochastic volatility by general compound Poisson processes and try to write the stochastic volatility in the following form:

$$
\frac{d \sigma^{2}\left(t, S_{t}\right)}{d t}=\gamma V+\frac{\alpha}{\tau}\left[\int_{t-\tau}^{t} \sigma\left(s, S_{s}\right) d W(s)+\int_{t-\tau}^{t} y_{s} d N(s)\right]^{2}-(\alpha+\gamma) \sigma^{2}\left(t, S_{t}\right)
$$


where $W(t)$ is a Brownian motion, $N(t)$ is a Poisson process with intensity $\lambda$, and $y_{t}$ is the jump size at time $t$. We assume that $\mathbb{E}\left[y_{t}\right]=A(t), \mathbb{E}\left[y_{s} y_{t}\right]=C(s, t), s<t$, and $\mathbb{E}\left[y_{t}^{2}\right]=B(t)=C(t, t)$ and $A(t), B(t)$, and $C(s, t)$ are all deterministic functions. Our purpose is to valuate variance swaps when the stochastic volatility satisfies this general equation. In order to get and check the results, we first consider two simple cases which are easier to model and implement but fundamental and still capture some characteristics of the market. In Section 4.1, we discuss the case that the jump size $y_{t}$ is always equal to constant one, that is, the jump part is represented by $\int_{t-\tau}^{t} d N(s)$, just simple Poisson processes. In Section 4.2, the jump part is still compound Poisson processes denoted as $\int_{t-\tau}^{t} y_{s} d N(s)$ but the jump size $y_{t}$ is assumed to be identically independent distributed random variable with mean value $\xi$ and variance $\eta$. The general case is discussed in Section 4.3. We can compare our results with the model in [14] and will see that it is a special case of our model after adding jumps in stochastic volatility. Finally, in Section 4.3 we will show that the model for stochastic volatility with delay and jumps keeps those good features of the model in [14].

\subsection{Simple Poisson Process Case}

We assume that the price of the underlying asset $S(t)$ follows the following stochastic delay differential equation (SDDE):

$$
d S(t)=\mu S(t) d t+\sigma\left(t, S_{t}\right) S(t) d W(t), \quad t>0,
$$

and the asset volatility is defined as the solution of the following equation:

$$
\frac{d \sigma^{2}\left(t, S_{t}\right)}{d t}=\gamma V+\frac{\alpha}{\tau}\left[\int_{t-\tau}^{t} \sigma\left(s, S_{s}\right) d W(s)+\int_{t-\tau}^{t} d N(s)\right]^{2}-(\alpha+\gamma) \sigma^{2}\left(t, S_{t}\right),
$$

where $W(t)$ is a Brownian motion and $N(t)$ is a Poisson process with intensity $\lambda$.

Recall that our purpose is to calculate $\mathbb{E}^{*}[v]=\mathbb{E}^{*}\left[\sigma^{2}\left(t, S_{t}\right)\right]$, the expectation of the variance under the risk-neutral measure. Since we assume that there is no Poisson process in the asset price, the change of measure is not different from the model we discussed in Section 3 to make it risk-neutral.

Let $\theta(t)=(\mu-r) / \sigma\left(t, S_{t}\right)$ be the market price of risk, which is adapted to the filtration $\mp(t)$, and, then by Girsanov's theorem for single Brownian motion, we obtain the risk-neutral probability measure which makes the discounted asset price a local martingale and $W^{*}(t)=$ $\int_{0}^{t} \theta(s) d s+W(t)$ is a Brownian motion under this probability measure.

Note that the change of measure does not change the Poisson intensity $\lambda$ since it is independent of the Brownian motion.

In the risk-neutral world, the volatility can be defined as follows:

$$
\frac{d \sigma^{2}\left(t, S_{t}\right)}{d t}=\gamma V+\frac{\alpha}{\tau}\left[\int_{t-\tau}^{t} \sigma\left(s, S_{s}\right) d W^{*}(s)+\int_{t-\tau}^{t} d N(s)-(\mu-r) \tau\right]^{2}-(\alpha+\gamma) \sigma^{2}\left(t, S_{t}\right)
$$


which can be expanded as follows:

$$
\begin{aligned}
& \frac{d \sigma^{2}\left(t, S_{t}\right)}{d t}=\gamma V+\frac{\alpha}{\tau}[\left(\int_{t-\tau}^{t} \sigma\left(s, S_{s}\right) d W^{*}(s)\right)^{2}+(N(t)-N(t-\tau))^{2}+(\mu-r)^{2} \tau^{2} \\
&+2\left(\int_{t-\tau}^{t} \sigma\left(s, S_{s}\right) d W^{*}(s)\right)(N(t)-N(t-\tau)) \\
&\left.-2\left(\int_{t-\tau}^{t} \sigma\left(s, S_{s}\right) d W^{*}(s)\right)(\mu-r) \tau-2(N(t)-N(t-\tau))(\mu-r) \tau\right] \\
&-(\alpha+\gamma) \sigma^{2}\left(t, S_{t}\right) .
\end{aligned}
$$

Now let us take the expectation under risk-neutral probability $\mathbb{P}^{*}$ on both sides of the equation. Note that the Brownian motion and the Poisson process are independent. Letting $v(t)=\mathbb{E}^{*}\left[\sigma^{2}\left(t, S_{t}\right)\right]$, we obtain the following deterministic delay differential equation:

$$
\begin{aligned}
& \frac{d v(t)}{d t}=\gamma V+\frac{\alpha}{\tau}\left[\mathbb{E}^{*}\left(\int_{t-\tau}^{t} \sigma\left(s, S_{s}\right) d W^{*}(s)\right)^{2}+\mathbb{E}^{*}((N(t)-N(t-\tau)))^{2}\right. \\
&\left.+(\mu-r)^{2} \tau^{2}-2 \mathbb{E}^{*}(N(t)-N(t-\tau))(\mu-r) \tau\right]-(\alpha+\gamma) v(t) \\
&=\gamma V+\frac{\alpha}{\tau}\left[\int_{t-\tau}^{t} v(s) d s+\operatorname{Var}^{*}(N(t)-N(t-\tau))+\left(\mathbb{E}^{*}(N(t)-N(t-\tau))\right)^{2}\right. \\
&\left.+(\mu-r)^{2} \tau^{2}-2 \mathbb{E}^{*}(N(t)-N(t-\tau))(\mu-r) \tau\right]-(\alpha+\gamma) v(t) \\
&=\gamma V+\alpha \lambda+\alpha \lambda^{2} \tau-2 \alpha \lambda \tau(\mu-r)+\alpha \tau(\mu-r)^{2}+\frac{\alpha}{\tau} \int_{t-\tau}^{t} v(s) d s-(\alpha+\gamma) v(t) .
\end{aligned}
$$

From this equation, if the intensity of the Poisson process $\lambda=0$, then it is the same as (3.8), the case of stochastic volatility with delay and without jumps.

Notice that (4.6) has a stationary solution

$$
v(t) \equiv X=V+\frac{\left[\alpha \lambda+\alpha \lambda^{2} \tau-2 \alpha \lambda \tau(\mu-r)+\alpha \tau(\mu-r)^{2}\right]}{\gamma}=V+\frac{\alpha}{\gamma}\left[\lambda+\tau(\lambda-\mu+r)^{2}\right] .
$$


Hence, the expectation of the realized variance or say the fair delivery price $K_{\mathrm{var}}$ of variance swap for stochastic volatility with delay and Poisson jump in stationary regime under risk-neutral measure $\mathbb{P}^{*}$ is equal to

$$
K_{\mathrm{var}}=\mathbb{E}^{*}[v]=\frac{1}{T} \int_{0}^{T} v(t) d t=V+\frac{\alpha}{\gamma}\left[\lambda+\tau(\lambda-\mu+r)^{2}\right]
$$

and the price $P$ of a variance swap at time $t$ given delivery price $K$ in this case should be

$$
P=e^{-r(T-t)}\left\{V-K+\frac{\alpha}{\gamma}\left[\lambda+\tau(\lambda-\mu+r)^{2}\right]\right\} \text {. }
$$

There is no way to write a solution in explicit form for arbitrarily given initial data. But we can understand an approximate behavior of solutions of (4.6) by looking at its eigenvalues. Let us substitute $v(t)=X+C e^{\rho t}$ into (4.6), where $X$ is defined in (4.7). Then, the characteristic equation for $\rho$ is

$$
\rho=\frac{\alpha}{\rho \tau}\left(1-e^{-\rho \tau}\right)-(\alpha+\gamma)
$$

which is equivalent to (when $\rho \neq 0$ )

$$
\rho^{2}=\frac{\alpha}{\tau}-\frac{\alpha}{\tau} e^{-\rho \tau}-(\alpha+\gamma) \rho
$$

The only solution to this equation is $\rho \approx-\gamma$, assuming that $\gamma$ is sufficiently small. Then the behavior of any solution is stable near $X$, and

$$
v(t)=X+C e^{-\gamma t}+O(t)
$$

or

$$
v(t) \approx X+C e^{-\gamma t}
$$

for large values of $t$. The approximation has the order $O(t)$, which follows from the solution of (4.10).

In this way, we have that

$$
v(t) \approx X+C e^{-\gamma t}=V+\frac{\alpha}{\gamma}\left[\lambda+\tau(\lambda-\mu+r)^{2}\right]+C e^{-\gamma t} .
$$

Since $v(0)=\sigma(0, S(0-\tau))=\sigma(0, \varphi(-\tau))=\sigma_{0}$, we can find the value of $C$ :

$$
C=\sigma_{0}^{2}-V-\frac{\alpha}{\gamma}\left[\lambda+\tau(\lambda-\mu+r)^{2}\right]
$$


Hence, the expectation of the realized variance or say the fair delivery price $K_{\mathrm{var}}$ of variance swap for stochastic volatility with delay and Poisson jump in general case under risk-neutral measure $\mathbb{P}^{*}$ is equal to

$$
K_{\mathrm{var}}=\mathbb{E}^{*}[v]=\frac{1}{T} \int_{0}^{T} v(t) d t \approx V+\frac{\alpha}{\gamma}\left[\lambda+\tau(\lambda-\mu+r)^{2}\right]+C \frac{1-e^{-\gamma T}}{\gamma T},
$$

where $C$ is given by (4.15).

Of course, (4.16) can also be written as

$$
K_{\mathrm{var}} \approx X+\left(\sigma_{0}^{2}-X\right) \frac{1-e^{-\gamma T}}{\gamma T}
$$

and the price $P$ of a variance swap at time $t$ given delivery price $K$ in this case should be

$$
P \approx e^{-r(T-t)}\left[X-K+\left(\sigma_{0}^{2}-X\right) \frac{1-e^{-r T}}{r T}\right]
$$

where $X$ is given by (4.7).

Summarizing, we have the following result.

Theorem 4.1. Consider stock price satisfying (4.2) with stochastic volatility in (4.3). Under conditions (C1)-(C3) (Section 3$)$ the price of the variance swap at time $t$ given delivery price $K$ is

$$
P \approx e^{-r(T-t)}\left[X-K+\left(\sigma_{0}^{2}-X\right) \frac{1-e^{-\gamma T}}{\gamma T}\right] \text {, }
$$

where $X$ is given by (4.7).

\subsection{Compound Poisson Process Case}

In the section we will consider the jumps represented by a compound Poisson process, and since it allows the jumps size to be a random number but not always one in Poisson process, the model is more realistic. Our approach in the last section can be easily used in compound Poisson process case.

In the risk-neutral world, the volatility can be defined as follows:

$$
\frac{d \sigma^{2}\left(t, S_{t}\right)}{d t}=\gamma V+\frac{\alpha}{\tau}\left[\int_{t-\tau}^{t} \sigma\left(s, S_{s}\right) d W^{*}(s)+\int_{t-\tau}^{t} y_{s} d N(s)-(\mu-r) \tau\right]^{2}-(\alpha+\gamma) \sigma^{2}\left(t, S_{t}\right)
$$

where $W^{*}(t)$ is a Brownian motion, $N(t)$ is a Poisson process with intensity $\lambda$, and $y_{t}$ is the jump size at time $t$ which is identically independent normally distributed random variable. We assume that the mean of $y_{t}$ is $\xi$ and the variance of $y_{t}$ is $\eta$. Note that the Poisson intensity $\lambda$ and the jump size $y_{t}$ do not change in risk-neutral world since they are independent of the Brownian motion. 
Our first step is still taking the expectation under risk-neutral probability $\mathbb{P}^{*}$ on both sides of the equation. Note that the Brownian motion and the compound Poisson process are independent. Letting $v(t)=\mathbb{E}^{*}\left[\sigma^{2}\left(t, S_{t}\right)\right]$, we obtain the following equation:

$$
\begin{aligned}
\frac{d v(t)}{d t}=\gamma V+\frac{\alpha}{\tau}\left[\int_{t-\tau}^{t} v(s) d s+\operatorname{Var}^{*}\left(\sum_{t-\tau \leq s \leq t} y_{s}\right)+\left(\mathbb{E}^{*}\left(\sum_{t-\tau \leq s \leq t} y_{s}\right)\right)^{2}\right. \\
\left.+(\mu-r)^{2} \tau^{2}-2 \mathbb{E}^{*}\left(\sum_{t-\tau \leq s \leq t} y_{s}\right)(\mu-r) \tau\right]-(\alpha+\gamma) v(t) \\
=\gamma V+\alpha \lambda\left(\xi^{2}+\eta\right)+\alpha \lambda^{2} \tau \xi^{2}-2 \alpha \lambda \tau \xi(\mu-r)+\alpha \tau(\mu-r)^{2}+\frac{\alpha}{\tau} \int_{t-\tau}^{t} v(s) d s-(\alpha+\gamma) v(t) .
\end{aligned}
$$

From this equation, if $\xi=1$ and $\eta=0$, the compound Poisson process is just a Poisson process, and then (4.21) becomes

$$
\frac{d v(t)}{d t}=\gamma V+\alpha \lambda+\alpha \lambda^{2} \tau-2 \alpha \lambda \tau(\mu-r)+\alpha \tau(\mu-r)^{2}+\frac{\alpha}{\tau} \int_{t-\tau}^{t} v(s) d s-(\alpha+\gamma) v(t)
$$

which is the same as (3.8).

Equation (4.21) has a stationary solution

$$
\begin{aligned}
v(t) & \equiv X=V+\frac{\left[\alpha \lambda\left(\xi^{2}+\eta\right)+\alpha \lambda^{2} \tau \xi^{2}-2 \alpha \lambda \tau \xi(\mu-r)+\alpha \tau(\mu-r)^{2}\right]}{\gamma} \\
& =V+\frac{\alpha}{\gamma}\left[\lambda\left(\xi^{2}+\eta\right)+\tau(\lambda \xi-\mu+r)^{2}\right] .
\end{aligned}
$$

Hence, the expectation of the realized variance or say the fair delivery price $K_{\mathrm{var}}$ of variance swap for stochastic volatility with delay and compound Poisson jump in stationary regime under riskneutral measure $\mathbb{P}^{*}$ is equal to

$$
K_{\mathrm{var}}=\mathbb{E}^{*}[v]=\frac{1}{T} \int_{0}^{T} v(t) d t=V+\frac{\alpha}{\gamma}\left[\lambda\left(\xi^{2}+\eta\right)+\tau(\lambda \xi-\mu+r)^{2}\right],
$$

and the price $P$ of a variance swap at time $t$ given delivery price $K$ in this case should be

$$
P=e^{-r(T-t)}\left\{V-K+\frac{\alpha}{\gamma}\left[\lambda\left(\xi^{2}+\eta\right)+\tau(\lambda \xi-\mu+r)^{2}\right]\right\} .
$$

In general case, we substitute $v(t)=X+C e^{\rho t}$ in (4.21) where $X$ is defined in (4.23). Then the characteristic equation for $\rho$ is

$$
\rho=\frac{\alpha}{\rho \tau}\left(1-e^{-\rho \tau}\right)-(\alpha+\gamma)
$$

which is also the same as that in the last section. 

have,

Therefore, the only solution to this equation is $\rho \approx-\gamma$, and by the same method, we

$$
\begin{gathered}
v(t) \approx X+C e^{-\gamma t}=V+\frac{\alpha}{\gamma}\left[\lambda\left(\xi^{2}+\eta\right)+\tau(\lambda \xi-\mu+r)^{2}\right]+C e^{-\gamma t}, \\
C=\sigma_{0}^{2}-V-\frac{\alpha}{\gamma}\left[\lambda\left(\xi^{2}+\eta\right)+\tau(\lambda \xi-\mu+r)^{2}\right] .
\end{gathered}
$$

Hence, the expectation of the realized variance or say the fair delivery price $K_{\mathrm{var}}$ of variance swap for stochastic volatility with delay and compound Poisson jump in general case under riskneutral measure $\mathbb{P}^{*}$ is equal to

$$
K_{\mathrm{var}}=E^{*}[v]=\frac{1}{T} \int_{0}^{T} v(t) d t \approx V+\frac{\alpha}{\gamma}\left[\lambda\left(\xi^{2}+\eta\right)+\tau(\lambda \xi-\mu+r)^{2}\right]+C \frac{1-e^{-\gamma T}}{\gamma T}
$$

where $C$ is given by (4.28).

Of course, (4.29) can also be written as

$$
K_{\mathrm{var}} \approx X+\left(\sigma_{0}^{2}-X\right) \frac{1-e^{-\gamma T}}{\gamma T}
$$

and the price $P$ of a variance swap at time $t$ given delivery price $K$ in this case should be

$$
P \approx e^{-r(T-t)}\left[X-K+\left(\sigma_{0}^{2}-X\right) \frac{1-e^{-\gamma T}}{\gamma T}\right]
$$

where $X$ is given by (4.23).

Summarizing, we have the following result.

Theorem 4.2. Consider a stock price satisfying (4.2) with stochastic volatility in (4.20). If conditions (C1)-(C3) (Section 3) and the conditions for $y_{t}$ (see above) are satisfied, then the price $P$ of a variance swap at time t given delivery price $K$ in this case is

$$
P \approx e^{-r(T-t)}\left[X-K+\left(\sigma_{0}^{2}-X\right) \frac{1-e^{-r T}}{r T}\right]
$$

where $\mathrm{X}$ is given by (4.23).

Remark 4.3. It is interesting to see that when $\tau=0$, which means there is no delay in the model, we have that

$$
\mathbb{E}^{*}[v] \approx \frac{1-e^{-\gamma T}}{\gamma T}\left(\sigma_{0}^{2}-V-\frac{\alpha \lambda\left(\xi^{2}+\eta\right)}{\gamma}\right)+V+\frac{\alpha \lambda\left(\xi^{2}+\eta\right)}{\gamma}
$$




\subsection{More General Case}

In the previous section, we assume that the mean value and variance of the jump size $y_{t}$ in the compound Poisson process are constants. Now we consider a more general case in which they are deterministic functions. The approach used in this section is different from the previous ones, which is a more general method and can be applied to derive the same formulae in the previous simple cases.

In the risk-neutral world, the volatility still satisfies the following equation:

$$
\frac{d \sigma^{2}\left(t, S_{t}\right)}{d t}=\gamma V+\frac{\alpha}{\tau}\left[\int_{t-\tau}^{t} \sigma\left(s, S_{s}\right) d W^{*}(s)+\int_{t-\tau}^{t} y_{s} d N(s)-(\mu-r) \tau\right]^{2}-(\alpha+\gamma) \sigma^{2}\left(t, S_{t}\right)
$$

where $W^{*}(t)$ is a Brownian motion, $N(t)$ is a Poisson process with intensity $\lambda$, and $y_{t}$ is the jump size at time $t$. We assume that $\mathbb{E}\left[y_{t}\right]=A(t), \mathbb{E}\left[y_{s} y_{t}\right]=C(s, t), s<t$, and $\mathbb{E}\left[y_{t}^{2}\right]=B(t)=$ $C(t, t)$, where $A(t), B(t)$, and $C(s, t)$ are all deterministic functions. Note that the change of measure does not change the Poisson intensity $\lambda$ and the distribution of jump size $y_{t}$, since they are independent of the Brownian motion.

Let $v(t)=\mathbb{E}^{*}\left[\sigma^{2}\left(t, S_{t}\right)\right]$ and take the expectation under risk-neutral probability $\mathbb{P}^{*}$ on both sides of (4.34). Noting that the Brownian motion and the Poisson process are independent, we obtain the following equation:

$$
\begin{aligned}
\frac{d v(t)}{d t}=\gamma V+\frac{\alpha}{\tau} & {\left[\int_{t-\tau}^{t} v(s) d s+\mathbb{E}^{*}\left(\int_{t-\tau}^{t} y_{s} d N(s)\right)^{2}+(\mu-r)^{2} \tau^{2}\right.} \\
& \left.-2 \mathbb{E}^{*}\left(\int_{t-\tau}^{t} y_{s} d N(s)\right)(\mu-r) \tau\right]-(\alpha+\gamma) v(t) .
\end{aligned}
$$

In order to compute the two expectations in this equation, we first introduce two lemmas as follows (see [43]).

Lemma 4.4. Define $I(t)=\int_{0}^{t} y_{s} d(N(s)-\lambda s)$; then $I(t)$ is a martingale and $\mathbb{E} I(t)=0$.

Lemma 4.5. Define $I(t)=\int_{0}^{t} y_{s} d(N(s)-\lambda s)$; then $\mathbb{E} I^{2}(t)=\lambda \mathbb{E} \int_{0}^{t} y_{s}^{2} d s$.

Therefore,

$$
\begin{aligned}
\mathbb{E}^{*}\left(\int_{t-\tau}^{t} y_{s} d N(s)\right) & =\mathbb{E}^{*}\left(\int_{t-\tau}^{t} y_{s} d(N(s)-\lambda s)\right)+\mathbb{E}^{*}\left(\int_{t-\tau}^{t} y_{s} d \lambda s\right) \\
& =\lambda \mathbb{E}^{*}\left(\int_{t-\tau}^{t} y_{s} d s\right) \\
& =\lambda \int_{t-\tau}^{t} A(s) d s
\end{aligned}
$$




$$
\begin{aligned}
\mathbb{E}^{*}\left(\int_{t-\tau}^{t} y_{s} d N(s)\right)^{2} & =\mathbb{E}^{*}\left(\int_{t-\tau}^{t} y_{s} d(N(s)-\lambda s)\right)^{2}+\mathbb{E}^{*}\left(\int_{t-\tau}^{t} y_{s} d \lambda s\right)^{2} \\
& =\lambda \int_{t-\tau}^{t} \mathbb{E}^{*} y_{s}^{2} d s+\lambda^{2} \mathbb{E}^{*}\left(\int_{t-\tau}^{t} y_{s} d s\right)^{2} \\
& =\lambda \int_{t-\tau}^{t} B(s) d s+\lambda^{2} \mathbb{E}^{*}\left(\int_{t-\tau}^{t} y_{s} d s\right)^{2} .
\end{aligned}
$$

To compute $\mathbb{E}^{*}\left(\int_{t-\tau}^{t} y_{s} d s\right)^{2}$, we take the derivative of $\left(\int_{t-\tau}^{t} y_{s} d s\right)^{2}$ and then integrate it:

$$
\begin{aligned}
d\left(\int_{t-\tau}^{t} y_{s} d s\right)^{2} & =2 \int_{t-\tau}^{t} y_{s} d s\left(y_{t}-y_{t-\tau}\right) d t \\
& =2 \int_{t-\tau}^{t}\left(y_{s} y_{t}-y_{s} y_{t-\tau}\right) d s d t \\
\left(\int_{t-\tau}^{t} y_{s} d s\right)^{2} & =2 \int_{0}^{t} \int_{u-\tau}^{u}\left(y_{s} y_{u}-y_{s} y_{u-\tau}\right) d s d u+\left(\int_{-\tau}^{0} y_{s} d s\right)^{2} \\
& =2 \int_{0}^{t} \int_{u-\tau}^{u}\left(y_{s} y_{u}-y_{s} y_{u-\tau}\right) d s d u+\int_{-\tau}^{0} \int_{-\tau}^{0} y_{s} y_{u} d s d u
\end{aligned}
$$

Now take the expectation under risk-neutral probability, we have that

$$
\begin{aligned}
\mathbb{E}^{*}\left(\int_{t-\tau}^{t} y_{s} d s\right)^{2} & =2 \int_{0}^{t} \int_{u-\tau}^{u}(C(s, u)-C(s, u-\tau)) d s d u+\int_{-\tau}^{0} \int_{-\tau}^{0} C(s, u) d s d u \\
& =K(t, \tau)+G
\end{aligned}
$$

where $K(t, \tau)=2 \int_{0}^{t} \int_{u-\tau}^{u}(C(s, u)-C(s, u-\tau)) d s d u$ and $G=\int_{-\tau}^{0} \int_{-\tau}^{0} C(s, u) d s d u$.

Taking into account (4.36), (4.37), and (4.39), equation (4.35) becomes

$$
\begin{aligned}
\frac{d v(t)}{d t}=\gamma V+\frac{\alpha}{\tau}\left[\int_{t-\tau}^{t} v(s) d s+\lambda \int_{t-\tau}^{t} B(s) d s+\lambda^{2}(K(t, \tau)+G)\right. \\
\left.+(\mu-r)^{2} \tau^{2}-2 \lambda \tau(\mu-r) \int_{t-\tau}^{t} A(s) d s\right]-(\alpha+\gamma) v(t) .
\end{aligned}
$$

We can check that (4.21) in Section 4.2 is a special case of (4.40) with $A(t)=\mathbb{E}\left[y_{t}\right]=\xi$, $B(t)=\mathbb{E}\left[y_{t}^{2}\right]=\operatorname{Var}\left[y_{t}\right]+\left(\mathbb{E}\left[y_{t}\right]\right)^{2}=\eta+\xi^{2}$, and $C(s, t)=\mathbb{E}\left[y_{s} y_{t}\right]=\mathbb{E}\left[y_{s}\right] \mathbb{E}\left[y_{t}\right]=\xi^{2}$.

To get the expectation of the realized variance in the risk-neutral world $\mathbb{E}^{*}[v]$, we have to find a solution to (4.40), a nonhomogeneous integrodifferential equation with delay. 
International Journal of Stochastic Analysis

After taking the first derivative of this equation, we obtain

$$
v^{\prime \prime}(t)=\frac{\alpha}{\tau}[v(t)-v(t-\tau)]-(\alpha+\gamma) v^{\prime}(t)+h(t, \tau)
$$

where $h(t, \tau)=(\alpha / \tau)\left[\lambda(B(t)-B(t-\tau))+\lambda^{2} K^{\prime}(t, \tau)-2 \lambda \tau(\mu-r)(A(t)-A(t-\tau))\right]$. This is a second-order delay differential equation with constant coefficients, and so Laplace transform can be applied to find its solution with initial condition $v(t)=\sigma\left(t, S_{t}\right), t \in[-\tau, 0]$, which is already known (see [44] or [45]).

Let us denote the Laplace transform of a function $f(t)$ as

$$
\mathcal{L}\{f(t)\}=\int_{0}^{\infty} f(t) e^{-s t} d t
$$

and do the Laplace transform for (4.41):

$$
L\left\{v^{\prime \prime}(t)\right\}=\frac{\alpha}{\tau}[\mathcal{L}\{v(t)\}-\mathcal{L}\{v(t-\tau)\}]-(\alpha+\gamma) \mathcal{L}\left\{v^{\prime}(t)\right\}+\mathcal{L}\{h(t, \tau)\}
$$

By change of variable and the property of Laplace transform, (4.43) yields

$$
\begin{aligned}
{\left[s^{2}+\right.} & \left.(\alpha+\gamma) s-\frac{\alpha}{\tau}\left(1-e^{-s \tau}\right)\right] \mathcal{L}\{v(t)\} \\
& =v^{\prime}(0)+(s+\alpha+\gamma) v(0)-\frac{\alpha}{\tau} e^{-s \tau} \int_{-\tau}^{0} v(t) e^{-s t} d t+\mathcal{L}\{h(t, \tau)\} .
\end{aligned}
$$

The characteristic function of (4.41) is

$$
C(s)=s^{2}+(\alpha+\gamma) s-\frac{\alpha}{\tau}\left(1-e^{-s \tau}\right) \approx s^{2}+\gamma s .
$$

Therefore,

$$
\mathcal{L}\{v(t)\}=C^{-1}(s)\left[v^{\prime}(0)+(s+\alpha+\gamma) v(0)-\frac{\alpha}{\tau} e^{-s \tau} \int_{-\tau}^{0} v(t) e^{-s t} d t+\mathcal{L}\{h(t, \tau)\}\right] .
$$

Applying the inverse transform (see [45]), we have that

$$
\begin{aligned}
v(t) \approx & \frac{1-e^{-\gamma t}}{\gamma} v^{\prime}(0)+\left[\frac{\alpha}{\gamma}\left(1-e^{-\gamma t}\right)+1\right] v(0)-\frac{\alpha}{\gamma \tau} \int_{-\tau}^{0} v(s)\left[1-e^{-\gamma(t-s-\tau)}\right] d s \\
& +\frac{1}{\gamma} \int_{0}^{t} h(s, \tau)\left[1-e^{-\gamma(t-s)}\right] d s+C .
\end{aligned}
$$


By the initial condition,

$$
C=\frac{\alpha}{\gamma \tau} \int_{-\tau}^{0} v(s)\left[1-e^{\gamma(s+\tau)}\right] d s
$$

Hence, the expectation of the realized variance or say the fair delivery price $K_{\mathrm{var}}$ of variance swap for stochastic volatility with delay and compound Poisson jump under risk-neutral measure $\mathbb{P}^{*}$ can be obtained by

$$
K_{\mathrm{var}}=\mathbb{E}^{*}[v]=\frac{1}{T} \int_{0}^{T} v(t) d t
$$

and the price $P$ of a variance swap at time $t$ given delivery price $K$ in this case should be

$$
P=e^{-r(T-t)}\left\{\mathbb{E}^{*}[v]-K\right\} .
$$

Of course, (4.41) can also be solved numerically.

Summarizing, we have the following result.

Theorem 4.6. Consider a stock price satisfying (4.2) with stochastic volatility in (4.34). If conditions (C1)-(C3) (Section 3) and the conditions for $y_{t}$ (see above) are satisfied, then the price P of a variance swap at time t given delivery price $K$ in this case is

$$
P=e^{-r(T-t)}\left\{\mathbb{E}^{*}[v]-K\right\},
$$

where $\mathbb{E}^{*}[v]$ is defined in (4.49) and $v(t)$ is defined in (4.47).

Remark 4.7. From expression (4.49), we note that only a double integral needs to be solved to get the expectation of the realized variance. If the functions $A(t), B(t)$, and $C(s, t)$ are in simple forms, say any integrable functions, the integral can even be solved explicitly. If we only consider the simple case in Sections 4.1 or 4.2 , the final analytical formulae only need algebraic computation and no numerical approximation is needed. Thus, we can say that the stochastic volatility model with delay and jumps is easy to implement and time saving.

\section{Delay as a Measure of Risk}

Volatility is the standard deviation of the change in value of a financial instrument with specific time horizon. It is often used to quantify the risk of the instrument over that time period. The higher the volatility is, the riskier the security is. The variance is a square of volatility and is also a measure of risk of a financial instrument. In this section we look at the estimation for variance through the delay to minimize the risk.

The compound Poisson case in Section 4.2 is a special case of the model we discussed in Section 4.3, but it is more basic and also shows some important characteristics. Therefore, in this section, we focus on this case. 
By Section 4.2, we obtain

$$
\begin{aligned}
E^{*}[v] \approx & V+\frac{\alpha}{\gamma}\left[\lambda\left(\xi^{2}+\eta\right)+\tau(\lambda \xi-\mu+r)^{2}\right] \\
& +\left\{\sigma_{0}^{2}-V-\frac{\alpha}{\gamma}\left[\lambda\left(\xi^{2}+\eta\right)+\tau(\lambda \xi-\mu+r)^{2}\right]\right\} \frac{1-e^{-\gamma T}}{\gamma T} .
\end{aligned}
$$

This expression contains all the information about our model since it contains all the initial parameters. We note that $\sigma_{0}^{2}=\sigma^{2}(0, \varphi(-\tau))$. So the sign of the second term in (5.1) depends on the relationship between $\sigma_{0}^{2}$ and $V+(\alpha / \gamma)\left[\lambda\left(\xi^{2}+\eta\right)+\tau(\lambda \xi-\mu+r)^{2}\right]$.

If $\sigma_{0}^{2}>V+(\alpha / \gamma)\left[\lambda\left(\xi^{2}+\eta\right)+\tau(\lambda \xi-\mu+r)^{2}\right]$, the second term in (5.1) is positive and $\mathbb{E}^{*}[v]$ stays above $V+(\alpha / \gamma)\left[\lambda\left(\xi^{2}+\eta\right)+\tau(\lambda \xi-\mu+r)^{2}\right]$, which means the risk is high.

If $\sigma_{0}^{2}<V+(\alpha / \gamma)\left[\lambda\left(\xi^{2}+\eta\right)+\tau(\lambda \xi-\mu+r)^{2}\right]$, the second term in (5.1) is negative and $\mathbb{E}^{*}[v]$ stays below $V+(\alpha / \gamma)\left[\lambda\left(\xi^{2}+\eta\right)+\tau(\lambda \xi-\mu+r)^{2}\right]$, which means the risk is low.

Therefore,

$$
\sigma_{0}^{2}=V+\frac{\alpha}{\gamma}\left[\lambda\left(\xi^{2}+\eta\right)+\tau(\lambda \xi-\mu+r)^{2}\right]
$$

defines the measure of risk in the stochastic volatility model with delay and jumps.

To reduce the risk we need to take into account the following relationship with respect to the delay $\tau$ (which follows from (5.1)):

$$
\tau>\frac{\left(\sigma_{0}^{2}-V\right) r-\alpha \lambda\left(\xi^{2}+\eta\right)}{\alpha(\lambda \xi-\mu+r)^{2}} .
$$

In this case, there is a way to control the delay.

\section{Numerical Example}

In this section, we apply the analytical solutions from Section 4.2 to price the variance swap of the S\&P60 Canada index for five years (January 1998-February 2002) (see [46]).

In the end of February 2002, we wanted to price the fixed leg of a variance swap based on the $S \& P 60$ Canada index. The statistics on log-returns of $S \& P 60$ Canada index for 5 years (January 1997-February 2002) is presented in Table 1.

From the histogram of the $S \& P 60$ Canada index log-returns on a 5-year historical period (1,300 observations from January 1998 to February 2002) leptokurtosis may be seen in the histogram. If we take a look at the graph of the $S \& P 60$ Canada index log-returns on a 5-year historical period we may see volatility clustering in the returns series. These facts indicate the conditional heteroscedasticity.

There are several parameters which need to be estimated from the data, the jump intensity $\lambda$, the mean value $\xi$, and the variance $\eta$ of the jump size. We use the following method to detect the jumps. If the difference between the spot log-return and the mean of our log-return series is larger than triple of the standard deviation, then we say that a jump occurs 
Table 1: Statistics on log-returns of S\&P60 Canada index.

\begin{tabular}{lc}
\hline Series & Log-returns of S\&P60 Canada index \\
\hline Sample & 11300 \\
Observations & 1300 \\
Mean & 0.000235 \\
Median & 0.000593 \\
Maximum & 0.051983 \\
Minimum & -0.101108 \\
Std. Dev. & 0.013567 \\
Skewness & -0.665741 \\
Kurtosis & 7.787327 \\
\hline
\end{tabular}

Table 2: Estimation of the GARCH(1,1) process.

Dependent variable: log-returns of S\&P60 Canada index prices

Method: ML-ARCH

Included observations: 1300

Convergence achieved after 28 observations

\begin{tabular}{lcccc}
\hline C & Coefficient & Std. error & Z-statistic & Prob. \\
\hline C & 0.000617 & 0.000338 & 1.824378 & 0.0681 \\
\hline C & \multicolumn{4}{c}{ Variance equation } \\
ARCH(1) & $2.58 E-06$ & $3.91 E-07$ & 6.597337 & 0 \\
GARCH(1) & 0.060445 & 0.007336 & 8.238968 & 0 \\
R-squared & 0.927264 & 0.006554 & 141.4812 & 0 \\
Adjusted R-squared & -0.000791 & Mean dependent var & - & 0.000235 \\
S.E. of regression & -0.003108 & S.D. dependent var & - & 0.013567 \\
Sum squared resid & 0.013588 & Akaike info criterion & - & -5.928474 \\
Log-likelihood & 0.239283 & Schwartz criterion & - & -5.912566 \\
\hline
\end{tabular}

at that time point (see [47]). Clewlow and Strickland [47] estimated jumps in asset price. In our case, the volatility is a trading instrument and can be considered as an asset. In this way, we can apply here their methodology. We count the number of the jumps, denoted as $N$, and the estimation of jump intensity $\bar{\lambda}=N / 1300$. The jump size is defined as the difference between the log-returns at a jump time point and the previous time point, and the sample mean and variance of these data are unbiased estimation of $\xi$ and $\eta$.

From the data in Table 1, we get the following estimation: $N=15, \lambda=N / 1300=$ $0.0115, \xi=-0.003$, and $\eta=0.0035$.

A GARCH $(1,1)$ regression is applied to the series, and the results are obtained as in Table 2. This table allows to generate different input variables to the volatility swap model. 
International Journal of Stochastic Analysis

We use the following relationship:

$$
\theta=\frac{V}{d t}, \quad k=\frac{1-\alpha-\beta}{d t},
$$

to calculate the following discrete $\operatorname{GARCH}(1,1)$ parameters:

$\operatorname{ARCH}(1,1)$ coefficient $\alpha=0.060445$;

$\operatorname{GARCH}(1,1)$ coefficient $\beta=0.927264$;

GARCH $(1,1)$ coefficient $\gamma=0.012391$;

the Pearson kurtosis (fourth moment of the drift-adjusted stock return) $\varepsilon=$ 7.787327;

long volatility $\theta=0.05289724$;

$k=3.09733$;

a short volatility $\sigma_{0}$ equals to 0.01 ;

$\mu=0.000235$;

$r=0.02$ and $\tau=1$ (day).

Parameter $(v)$ may be found from the expression $V=C /(1-\alpha-\beta)$, where $C=2.58 \times 10^{-6}$ is defined in Table 2. Thus, $V=0.00020991$ and

$$
d t=\frac{1}{252}=0.003968254 .
$$

Now, applying the analytical solutions (4.29) for a variance swap maturity $T$ of 1 year, we find the following value:

$$
\begin{aligned}
& V+\frac{\alpha}{\gamma}\left[\lambda\left(\xi^{2}+\eta\right)+\tau(\lambda \xi-\mu+r)^{2}\right] \\
& =0.0002+\frac{0.0604}{0.0124} \times\left[0.0115 \times\left[(-0.003)^{2}+0.0035\right]\right. \\
& \left.+(0.0115 \times(-0.003)-0.0002+0.0124)^{2}\right] \\
& =0.0023, \\
& \mathbb{E}^{*}[v] \approx V+\frac{\alpha}{\gamma}\left[\lambda\left(\xi^{2}+\eta\right)+\tau(\lambda \xi-\mu+r)^{2}\right] \\
& +\left\{\sigma_{0}^{2}-V-\frac{\alpha}{\gamma}\left[\lambda\left(\xi^{2}+\eta\right)+\tau(\lambda \xi-\mu+r)^{2}\right]\right\} \frac{1-e^{-\gamma T}}{\gamma T} \\
& =0.0023+(0.0001-0.0023) \times \frac{1-e^{-0.0124}}{0.0124} \\
& =0.0001136 \text {. }
\end{aligned}
$$




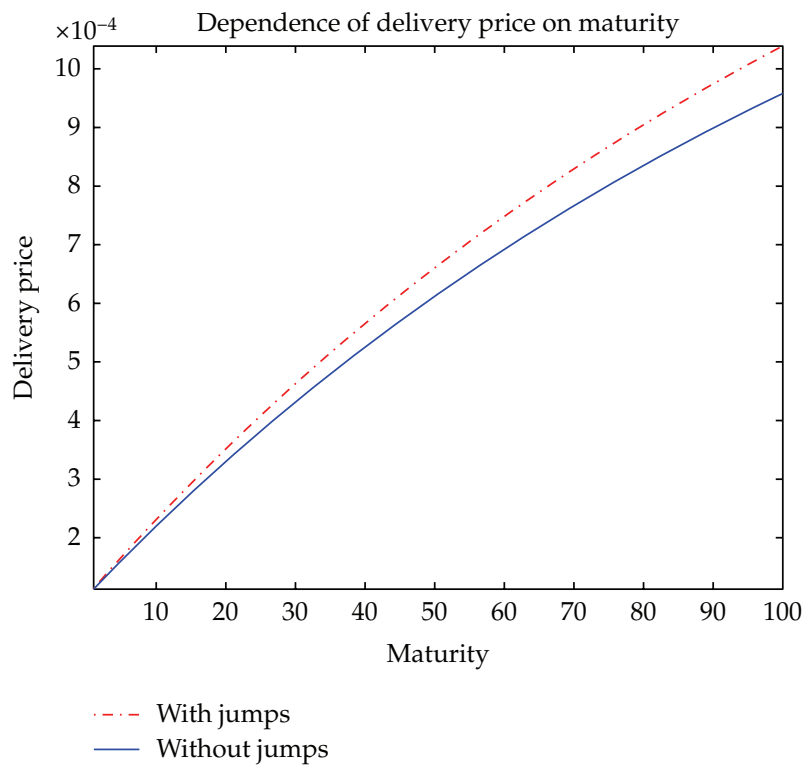

Figure 1: Dependence of delivery price on maturity (S\&P60 Canada index).

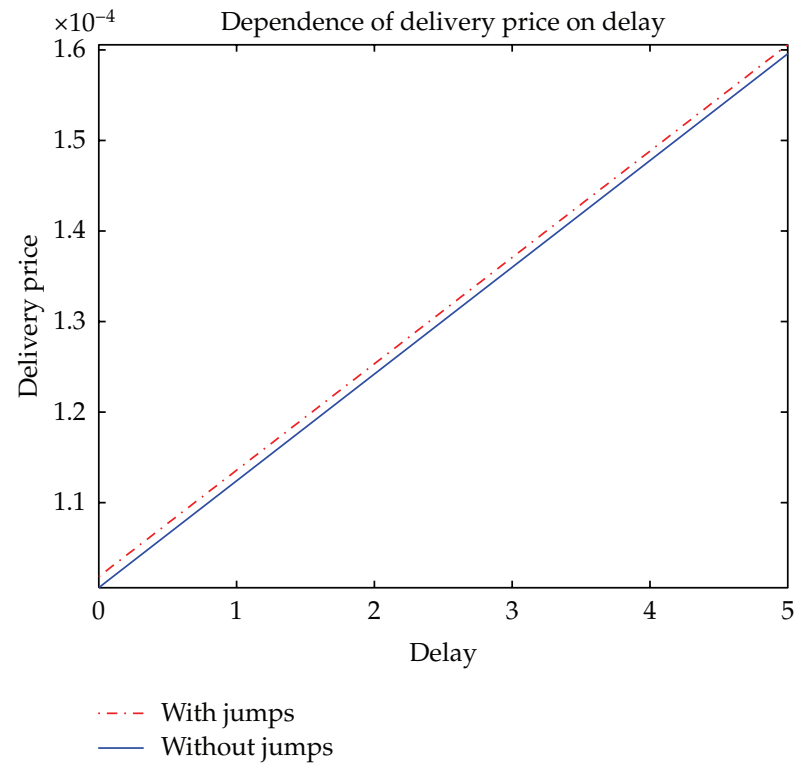

Figure 2: Dependence of delivery price on delay (S\&P60 Canada index).

Repeating this approach for a series of delays up to 5 days and series of maturities up to 100 years, we compare our results with the no jump model and obtain the following plot (see the appendix, Figures 1 and 2) of S\&P60 Canada index variance swap. Figure 3 (see the appendix) depicts the dependence of delivery price of variance swaps on jump intensity. Figures 4, 5, and 6 (see the appendix) depict the dependence of delivery price of variance swaps on two variables of maturity, delay, and jump intensity. 


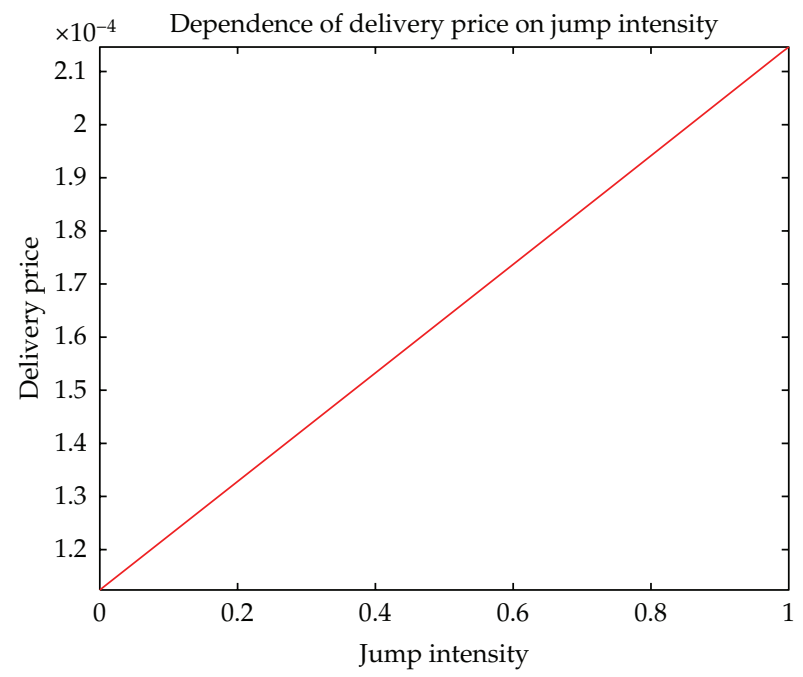

Figure 3: Dependence of delivery price on jump intensity (S\&P60 Canada index).

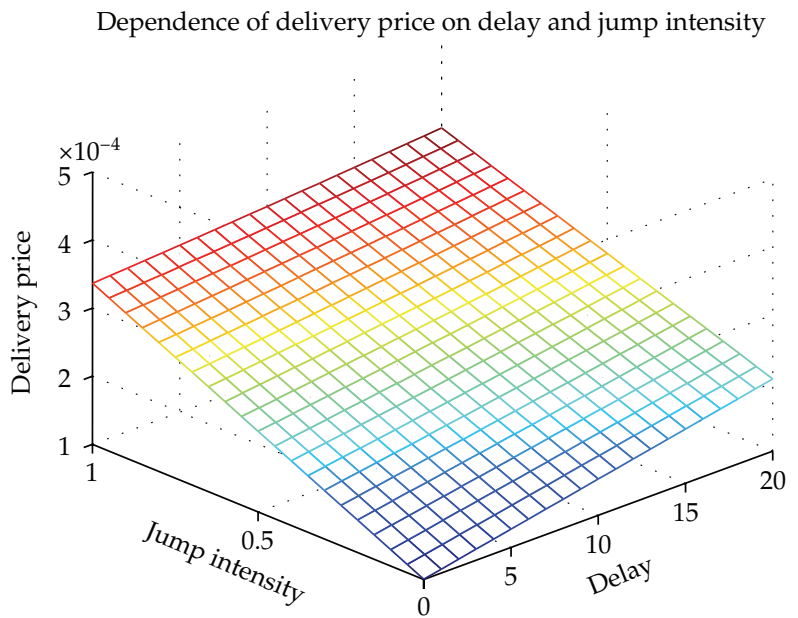

Figure 4: Dependence of delivery price on delay and jump intensity (S\&P60 Canada index).

\section{An Important Remark with Respect to Our Model with Jumps}

We note that we provided back testing in [48] to show that estimated variance swap prices are close to realized prices for stochastic volatility without jumps. Figure 1 shows that, in presence of jumps, the estimated delivery prices are close for models with and without jumps (the order of estimation is $10^{-4}$ ). This marginal difference between the delivery prices $\mathbb{E}^{*}[v]$ with and without jumps is because our $\lambda$ is very small since we calculated the $\lambda=0.0115$ with respect to the number of jumps, 15, divided by the number of samples, 1300. We count one jump if the price change is bigger than 3 times the standard deviation. For example, 


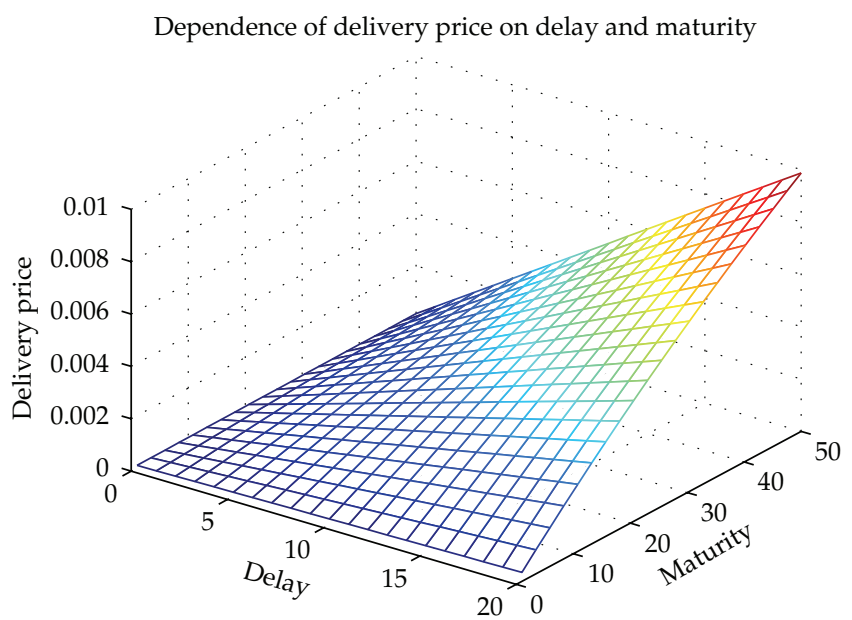

Figure 5: Dependence of delivery price on delay and maturity (S\&P60 Canada index).

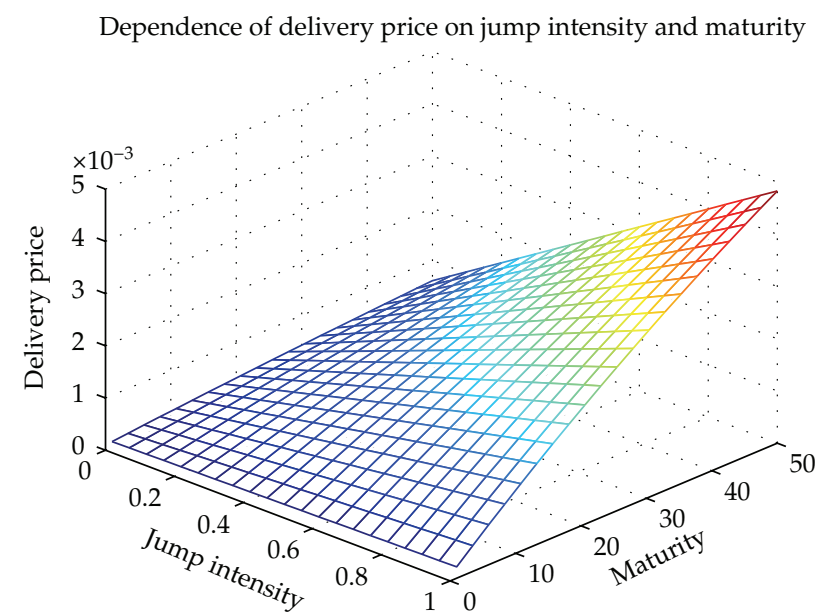

Figure 6: Dependence of delivery price on jump intensity and maturity (S\&P60 Canada index).

without jumps, $\mathbb{E}^{*}[v]=0.0001124$, and, with jumps $(\lambda=0.0115), \mathbb{E}^{*}[v]=0.00011365$ (our case). In this way, the relative change with respect to no jumps is $1.07 \%$. If we increase the jump intensity to, for example, $\lambda=0.1$, then $\mathbb{E}^{*}[v]=0.0001233$ (see (4.29) for calculation), and the relative change with respect to no jumps is $9.7 \%$. For $\lambda=0.2$ the relative change becomes $19.4 \%$, and for $\lambda=0.5$ the relative change would be $48.6 \%$ ! All in all, in our case, with $\lambda=0.0115$, even $1 \%$ change cannot be ignored in the market, because our value for $\mathbb{E}^{*}[v]$ is just for one unit of expected variance. But if one buys many contracts and times a notional amount $N$, then $1 \%$ change is not that small in value anymore. 


\section{Conclusion}

In this paper we studied stochastic volatility model with delay and jumps to price variance swaps. We applied a general approach to derive the analytical close forms for expectation of the realized continuously sampled variance for stochastic volatility with delay and jumps. The jump part in our model is represented by a general version of compound Poisson processes. The key features of the model are the following: (i) continuous-time analogue of discrete-time GARCH model, (ii) mean reversion, (iii) containing the same source of randomness as stock price, (iv) completeness of the market, (v) incorporating the expectation of log-return, and (vi) incorporating the jumps in volatility. The model is also easy to implement and time saving. Besides, we presented a lower bound for delay as a measure of risk. From the numerical example, we found that after adding jumps in volatility, the expectation of the realized variance is higher than the one without jumps for variance swaps. It is easy to explain it since the existence of jumps means the market is more risky which asks for higher cost of variance swaps.

For further study, we may add jumps in the spot price of the underlying asset which is also an important characteristic found in real markets. Besides, the jump part can be in more complicated form, for example, $\int_{t-\tau}^{t} \sigma\left(s, S_{s}\right) d N(s)$. We may also consider applying our methods to price volatility swaps which make more sense for investors to hedge volatility. Also, one of the referees suggested considering a continuum of delay times, not only just one delay time, and an associated "memory function" to weight their contributions which would make more sense for introduced model. However, all these topics are very interesting but are beyond the scope of the present paper and will be considered in our future research work.

\section{Appendix}

\section{Figures}

Figure 1 depicts the dependence of delivery price on maturity for $S \& P 60$ Canada index (1998-2002) for stochastic volatility (SV) with and without jumps. As we can see the delivery price for SV with jumps is higher than that for SV without jumps because the security in this case is riskier.

Figure 2 depicts the dependence of delivery price on delay for $S \& P 60$ Canada index (1998-2002) for SV with and without jumps. Again, the price is obviously higher. We note that, if the delay is zero, then the price approaches the constant value when maturity $T$ is large (i.e., see formula (4.36)). If the delay is not zero, then the delivery price is proportional to the length of delay.

Figure 3 depicts the dependence of delivery price on jump intensity for S\&P60 Canada index (1998-2002) for SV with jumps. The higher the intensity of jumps is, the higher the delivery price is.

Figure 4 depicts the dependence of delivery price on delay and jump intensity for S\&P60 Canada index (1998-2002). This figure is a combination of Figures 2 and 3 for SV with jumps and delay.

Figure 5 depicts the dependence of delivery price on delay and maturity for $S \& P 60$ Canada index (1998-2002). This figure is a combination of Figures 1 and 2 for SV with jumps and delay.

Figure 6 depicts the dependence of delivery price on jump intensity and maturity for S\&P60 Canada index (1998-2002). This figure is a combination of Figures 1 and 3 for SV with jumps and delay. 


\section{Acknowledgments}

The research is supported by NSERC Grant RPG 312593. The authors would like to thank an anonymous referee very much for valuable comments and suggections that improved the present paper. The authors remain responsible for any errors in this paper.

\section{References}

[1] P. Carr and D. Madan, Towards a Theory of Volatility Trading, 1998.

[2] K. Demeterfi, E. Derman, M. Kamal, and J. Zou, "A guide to volatility and variance swaps," The Journal of Derivatives, vol. 6, pp. 9-32, 1999.

[3] S. Heston and S. Nandi, "Derivatives on volatility: some simple solutions based on observables," Tech. Rep. 2000-20, Federal Reserve Bank of Atlanta, Atlanta, Ga, USA, 2000.

[4] O. Brockhaus and D. Long, "Volatility swaps made simple," Risk, pp. 92-96, 2000.

[5] A. Javaheri, P. Wilmott, and E. Haug, "GARCH and volatility swaps," Quantitative Finance, vol. 4, no. 5, pp. 589-595, 2004.

[6] S.-P. Zhu and G.-H. Lian, "Pricing variance swaps with stochastic volatility," in Proceedings of the World Congress on Engineering, vol. 2, pp. 1359-1364, July 2009.

[7] S.-P. Zhu and G.-H. Lian, "A closed-form exact solution approach for pricing variance swaps with stochastic volatility," Mathematical Finance, vol. 21, no. 2, pp. 233-256, 2011.

[8] M. Broadie and A. Jain, "The effect of jumps and discrete sampling on volatility and variance swaps," International Journal of Theoretical and Applied Finance, vol. 11, no. 8, pp. 761-797, 2008.

[9] F. Black and M. Scholes, "The pricing of options and corporate liabilities," The Journal of Political Economy, vol. 81, pp. 637-654, 1973.

[10] J. Hull and A. White, "The pricing of options on assets with stochastic volatilities," Journal of Finance, vol. 42, pp. 281-300, 1987.

[11] S. Heston, "A closed-form solution for options with stochastic volatility with applications to bond and currency options," Review of Financial Studies, vol. 6, pp. 327-343, 1993.

[12] Y. Kazmerchuk, A. Swishchuk, and J. Wu, "A continuous-time Garch model for stochastic volatility with delay," Canadian Applied Mathematics Quarterly, vol. 13, no. 2, pp. 123-149, 2005.

[13] Y. Kazmerchuk, A. Swishchuk, and J. Wu, "The pricing of options for securities markets with delayed response," Mathematics and Computers in Simulation, vol. 75, no. 3-4, pp. 69-79, 2007.

[14] A. Swishchuk, "Modeling and pricing of variance swaps for stochastic volatilities with delay," WILMOTT Magazine, no. 19, pp. 63-73, 2005.

[15] A. Swishchuk, "Modelling and pricing of variance swaps for multi-factor stochastic volatilities with delay," Canadian Applied Mathematics Quarterly, vol. 14, no. 4, pp. 439-467, 2006.

[16] J. A. Scheinkman and B. LeBaron, "Nonlinear dynamics and stock returns," Journal of Business, vol. 62, pp. 311-337, 1989.

[17] V. Akgiray, "Conditional heteroscedasticity in time series of stock returns: evidence and forecasts," Journal of Business, vol. 62, no. 1, pp. 55-80, 1989.

[18] P. Kind, R. Sh. Liptser, and W. J. Runggaldier, "Diffusion approximation in past dependent models and applications to option pricing," The Annals of Applied Probability, vol. 1, no. 3, pp. 379-405, 1991.

[19] D. G. Hobson and L. C. G. Rogers, "Complete models with stochastic volatility," Mathematical Finance, vol. 8, no. 1, pp. 27-48, 1998.

[20] M.-H. Chang and R. K. Youree, "The European option with hereditary price structures: basic theory," Applied Mathematics and Computation, vol. 102, no. 2-3, pp. 279-296, 1999.

[21] M.-H. Chang and R. K. Youree, "The European option with hereditary price structures: basic theory," Applied Mathematics and Computation, vol. 102, no. 2-3, pp. 279-296, 1999.

[22] S. E. A. Mohammed, M. Arriojas, and Y. Pap, “A delayed black and scholes formula," . In press.

[23] T. Bollerslev, "Generalized autoregressive conditional heteroskedasticity," Journal of Econometrics, vol. 31, no. 3, pp. 307-327, 1986.

[24] J.-C. Duan, "The GARCH option pricing model," Mathematical Finance, vol. 5, no. 1, pp. 13-32, 1995.

[25] R. Cont and P. Tankov, Financial Modelling with Jump Processes, Chapman \& Hall/CRC, Boca Raton, Fla, USA, 2004.

[26] S. Howison, A. Rafailidis, and H. Rasmussen, "On the pricing and hedging of volatility derivatives," Applied Mathematical Finance, vol. 11, no. 4, pp. 317-348, 2004. 
[27] J. Muhle-Karbe, J. Kallsen, and M. Voss, "Pricing options on variance in affine stochastic volatility models," Mathematical Finance. In press.

[28] B. Eraker, M. Johannes, and N. Polson, "The impact of jumps in volatility and returns," Journal of Finance, vol. 58, no. 3, pp. 1269-1300, 2003.

[29] D. Duffie, J. Pan, and K. Singleton, “Transform analysis and asset pricing for affine jump-diffusions," Econometrica. Journal of the Econometric Society, vol. 68, no. 6, pp. 1343-1376, 2000.

[30] R. C. Merton, “Option pricing when underlying stock returns are discontinuous," Journal of Financial Economics, vol. 3, no. 1-2, pp. 125-144, 1976.

[31] D. S. Bates, "Jumps and stochastic volatility: exchange rate processes implicit in deutsche mark options," Review of Financial Studies, vol. 9, no. 1, pp. 69-107, 1996.

[32] G. Bakshi, C. Charles, and Z. Chen, "Empirical performance of alternative option pricing models," Journal of Finance, vol. 52, no. 5, pp. 2003-2049, 1997.

[33] M. Broadie, M. Chernov, and M. Johannes, "Model specification and risk premia: evidence from futures options," Journal of Finance, vol. 62, no. 3, pp. 1453-1490, 2007.

[34] J. Liu, F. A. Longstaff, and J. Pan, "Dynamic asset allocation with event risk," Journal of Finance, vol. 58, no. 1, pp. 231-359, 2003.

[35] J. Liu and J. Pan, "Dynamic derivative strategies," Journal of Financial Economics, vol. 69, no. 3, pp. 401-430, 2003.

[36] S. Dieckmann and M. Gallmeyer, "The equilibrium allocation of diffusive and jump risks with heterogeneous agents," Journal of Economic Dynamics E Control, vol. 29, no. 9, pp. 1547-1576, 2005.

[37] T. Bollerslev, T. H. Law, and G. Tauchen, "Risk, jumps, and diversification," Journal of Econometrics, vol. 144, no. 1, pp. 234-256, 2008.

[38] O. Barndorff-Nielsen and N. Shephard, "Variation, jumps, market frictions and hight frequency data in financial econometrics," in Proceedings of the 9th World Congress of the Econometric Society, 2005.

[39] Y. Aït-Sahalia and J. Jacod, "Estimating the degree of activity of jumps in high frequency data," The Annals of Statistics, vol. 37, no. 5A, pp. 2202-2244, 2009.

[40] V. Todorov and G. Tauchen, “Volatility jumps,” Tech. Rep., Durham, NC, USA, 2008.

[41] Y. Aït-Sahalia, "Testing continuous-time models of the spot interest rate," Review of Financial Studies, vol. 9, no. 2, pp. 385-426, 1996.

[42] Salah-E.A. Mohammed, "Stochastic differential systems with memory: theory, examples and applications," in Stochastic Analysis and Related Topics, VI, vol. 42, pp. 1-77, Birkhäuser, Boston, Mass, USA, 1998.

[43] D. Lamberton and B. Lapeyre, Introduction to Stochastic Calculus Applied to Finance, Chapman \& Hall, London, UK, 1996.

[44] J. K. Hale and S. M. Lunel, Introduction to Functional-Differential Equations, vol. 99 of Applied Mathematical Sciences, Springer, New York, NY, USA, 1993.

[45] R. Bellman and K. L. Cooke, Differential-Difference Equations, Academic Press, New York, NY, USA, 1963.

[46] L. Zabre, R. Theoret, and P. Rostan, "Pricing volatility swaps: empirical testing with canadian data," Tech. Rep. 17-2002, Centre de Recherche en Gestion, 2002.

[47] L. Clewlow and C. Strickland, Energy Derivatives: Pricing and Risk Management, Lacima Group, Houston, Tex, USA, 2000.

[48] A. Swishchuk, "Modeling of variance and volatility swaps for financial markets with stochastic volatilities," WILMOTT Magazine, no. 2, pp. 64-72, 2004. 


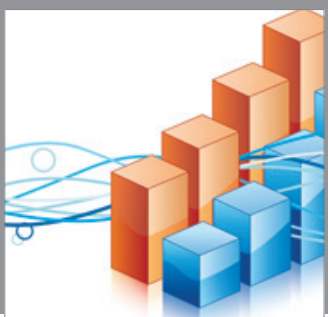

Advances in

Operations Research

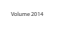

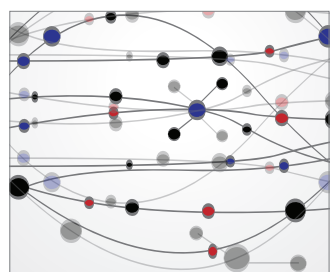

\section{The Scientific} World Journal
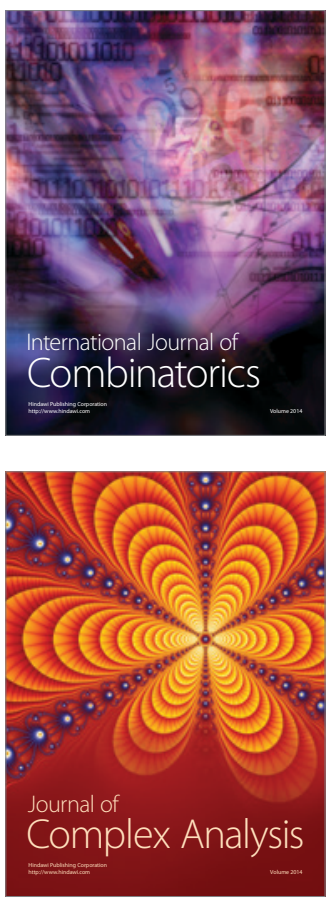

International Journal of

Mathematics and

Mathematical

Sciences
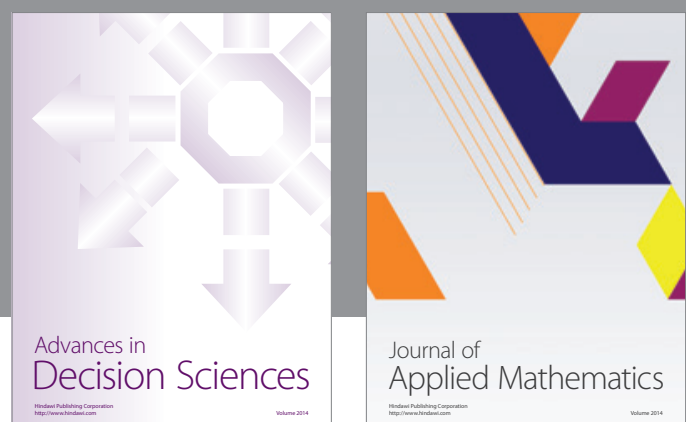

Journal of

Applied Mathematics
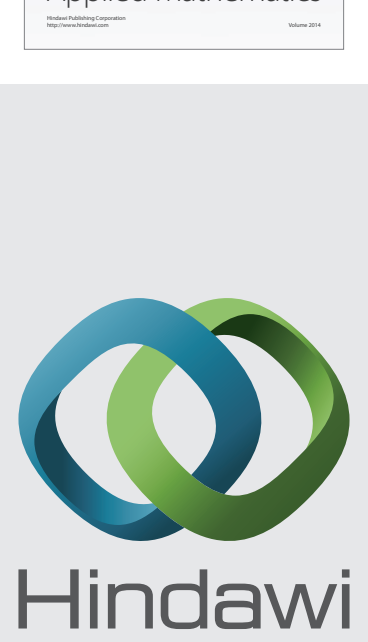

Submit your manuscripts at http://www.hindawi.com
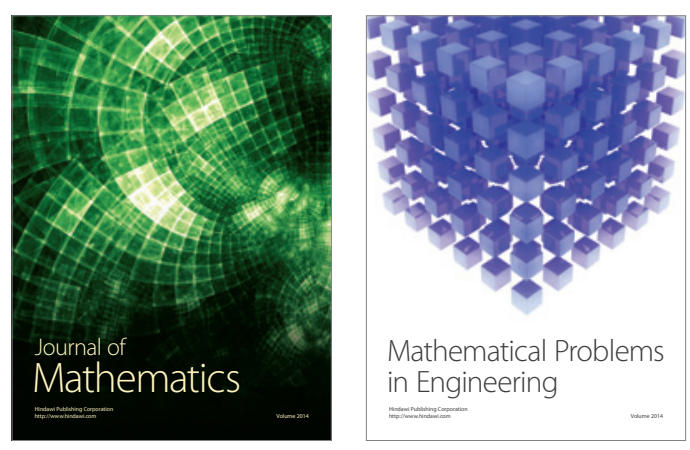

Mathematical Problems in Engineering
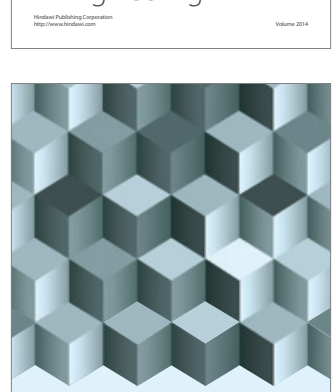

Journal of

Function Spaces
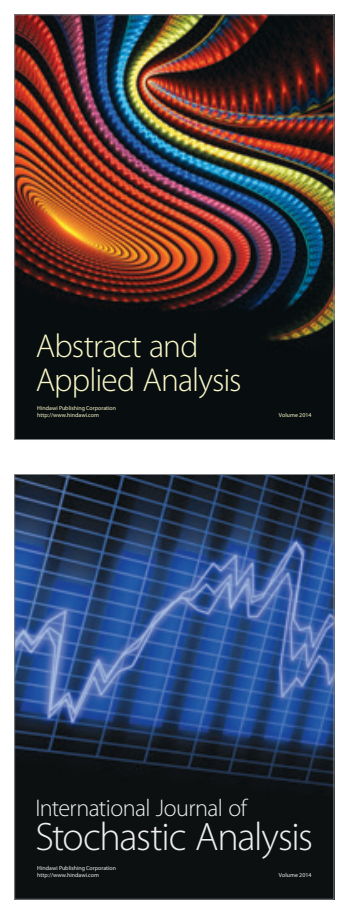

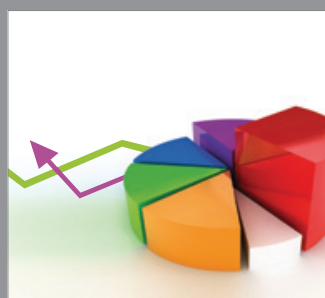

ournal of

Probability and Statistics

Promensencen
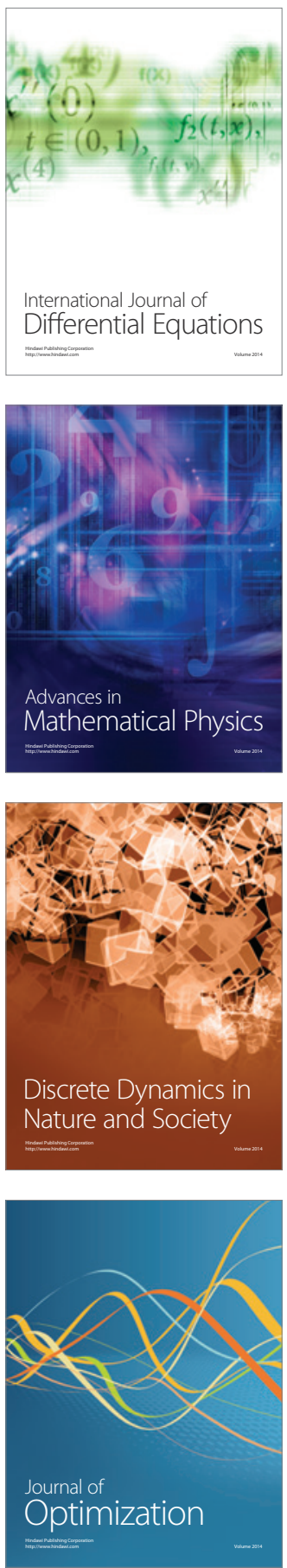